\title{
SATELLITE IMAGE CLASSIFICATION: FROM HANDCRAFTED FEATURES TO DEEP LEARNING FEATURES
}

\author{
Khushbu R. Joshi \\ Research Scholar, Faculty of Engineering and Technology, Sankalchand Patel University, \\ Visnagar, Gujarat 384315, India \\ Lecturer, EC Department, LDRP Institute of Technology Research, \\ Gandhinagar, Gujarat 382015, India \\ skhushi86@gmail.com \\ Manish I. Patel \\ Assistant Professor, Department of Electronics and Communication Engineering, Institute of Technology, \\ Nirma University \\ Ahmedabad, Gujarat 382481, India \\ manish.i.patel@nirmauni.ac.in
}

\begin{abstract}
Every day, satellites capture huge volumes of data and monitor the earth's surface. This is a timeconsuming process to manually classify the images into their appropriate classes. A classification system that automatically classifies the images into the proper classes is required. In this article, we have used handcrafted features and deep learning features to classify the satellite images. As handcrafted features, HLAC features have been used and for deep learning features ResNet-50 and ResNet-101 models have been used. This article provides a method for detecting HLAC features in multi-class satellite images and automatically classifying them into their appropriate classes. To classify an image, the image's features are first extracted, and then classification is performed utilizing those features. An SVM classifier is used to classify the images. The Sentinel-2 satellite images from the EuroSAT dataset were used. The accuracy and F1-score were used to assess the performance of the classifier. The SVM classifier with HLAC feature extraction had an F1-score of $\mathbf{8 6 . 2 6}$ and an accuracy of $\mathbf{8 9 . 3 5 \%}$. The dataset's classification accuracy was also assessed using a deep learning model. We obtained a $98.69 \%$ accuracy using deep learning models which is $0.12 \%$ higher than the benchmarked.
\end{abstract}

Keywords: Image classification; feature extraction; support vector machine; deep learning.

\section{Introduction}

A computer vision system is a type of technology that identifies and detects features of a specific class in digital images and videos. Many computer vision applications, such as image matching and classification, rely on local feature detection and description. The performance of the local feature detectors and descriptors utilized in the approaches determines the accuracy of these applications.

With the advancement of machine learning and deep learning approaches in recent decades, new algorithms and methodologies have been introduced. When enough data is provided, machine learning algorithms can take the work to the next level. Deep learning algorithms are capable of processing vast amounts of data. However, this may generate concerns in the mind of a researcher about selecting the appropriate algorithm and method for a given application to improve performance. The algorithms chosen are mostly determined by the type of application and the amount of data to be processed.

Satellites monitor the earth's surface and collect massive amounts of data daily. Satellite images are available in a wide range of bands and classes. An automatic classification system that automatically recognizes photos based on their class was required to deal with huge amounts of data. Classification techniques can be used to extract important information from an image. Image feature classification is a classification technique that uses extracted features such as spatial and spectral characteristics to classify images. 
There are two types of machine learning approaches: supervised and unsupervised learning. A supervised learning method relies on past data knowledge. Unsupervised learning, on the other hand, does not require any prior data knowledge. The approaches can also be classified as parametric and non-parametric methods.

The decision in parametric approaches is based on a probability density function, such as a Gaussian distribution, and for each class, a function may be constructed using the mean vector and covariance matrix. The Naive Bayes model and logistic regression are two examples of this strategy.

Non-parametric approaches, on the other hand, are not based on any probability distributions or assumptions. kNN, Decision tree (DT) [Quinlan (1986)], and other methods are examples of this method. Efforts to improve performance have been recorded in the literature, and research is still underway. In this work, HLAC features were used with SVM to improve accuracy.

The following are some of the contributions made by this research:

This study has been carried out to study the performance evaluation of handcrafted features and deep learning features.

1. To evaluate the performance of the handcrafted features, HLAC features were used to extract the important features from the satellite images of the EuroSAT dataset. The most primary usage of SVM is as a binary classifier. The classifier's performance for multi-class classification is evaluated using the SVM for the multiclass (one-vs-all) approach.

2. Deep learning models are used to assess the dataset's performance. We employed pre-trained ResNet-50 and ResNet-101 models and trained on the EuroSAT dataset, which produced better results than benchmarks.

The following is a breakdown of the paper's structure. The relevant work is displayed in Section 2. The proposed technique is described in Section 3. Section 4 represents the feature extraction using the deep learning method. Section 5 presents the findings and comments. Section 6 concludes with a summary of previous work as well as expectations.

\section{Relevant Work}

One of the most crucial aspects of any classification system is feature extraction from images. This section details the literature review undertaken by numerous key contributors to the project. Scale-Invariant Feature Transform (SIFT) [Lowe (2004)], Histograms of Oriented Gradients (HoG) [Dalal and Triggs, (2005)], and texture features [Haralick et al. (1973)] are all common feature descriptors. CNN-based approaches have recently received a lot of attention.

Liu et al. [Liu et al. (2020)] presented a DeepSat classification system based on "handcrafted" characteristics and CNN. The performance of CNN-based approaches is better, but they are also more complex. Gabriela et al. [Gabriela et al. (2018)] presented a comparison of handcrafted and deep learning-based features in the progression of local features from handcrafted to deep learning-based methods. They concluded that, while deep learning-based approaches are faster, more automatic, and have higher accuracy, their complexity grows as processing capacity increases.

Deep CNN models out performances in classification [Zhong et al. (2017), Oh \& Kang (2017) and HumeauHeurtier (2019)], data fusion [Kussul et al. (2016)] and object detection [Li et al. (2020), Cao et al. (2020)]. Deep learning approaches necessitate the utilization of GPU and TPU, as well as the complicated and timeconsuming training of the model. [Liu et al. (2020), Tianyu et al. (2018)] also discusses the concept of merging features. The use of $\mathrm{CNN}$ in conjunction with handcrafted features demonstrates that handcrafted features can outperform CNN.

Dewangkoro and Arymurthy [Dewangkoro and Arymurthy (2021)] used numerous CNNs for feature extraction, including VGG-19, ResNet-50, and Inception V3, as well as SVM and Twin SVM (TWSVM) as a classifier.

When the dataset isn't large enough and better computational power isn't available, machine learning algorithms based on SVM are shown to be a viable option. For land use/cover categorization, Przemysaw Kupidura [Kupidura (2019)] evaluated grey level co-occurrence matrix (GLCM) features, Laplace filters, and granulometric analysis. The results revealed that the efficacy of selected GLCM features and granulometric analysis were found to be greater for textural data. Texture analysis is more effective for high-resolution satellite photos. For land cover categorization, Noi and Kappas [Noi and Kappas (2018)] examined RF, kNN, and SVM classifiers and found that SVM had a better Overall All (OA) accuracy. 
Jivane and Soundrapandiyan [Jivane and Soundrapandiyan (2017)] used SURF and SVM to recognize oil tanks in satellite images. For linear multi-class classification of building photos, Varma and Rao [Varma and Rao (2017)] integrated generic techniques with SVM. Rana and Suryanarayana [Rana and Suryanarayana (2020)] established a novel technique for Sentinel-2 satellite imagery that tested MLE, RF, and SVM classification algorithms and discovered that SVM had the highest accuracy.

Satellite images may be efficiently classified using a variety of machine learning algorithms. SVM is a supervised machine learning approach for classification that is widely utilized. SVM has some kernel functions that transform data into higher dimensions, allowing features recognized and extracted from satellite pictures to be sorted into the appropriate classes. Satellite imagery is a type of texture. As a result, feature identification and extraction system were required that could efficiently extract texture features from satellite photos. SVM performed well with GLCM [Zhang et al. (2017)], auto-correlation [Hayashi and Uchida (2018)], and multichannel HLAC [Uehara (2017)] for texture feature extraction.

\section{Image Classification using Handcrafted Features: Higher Order Local Auto-Correlation (HLAC)}

\subsection{Proposed Method}

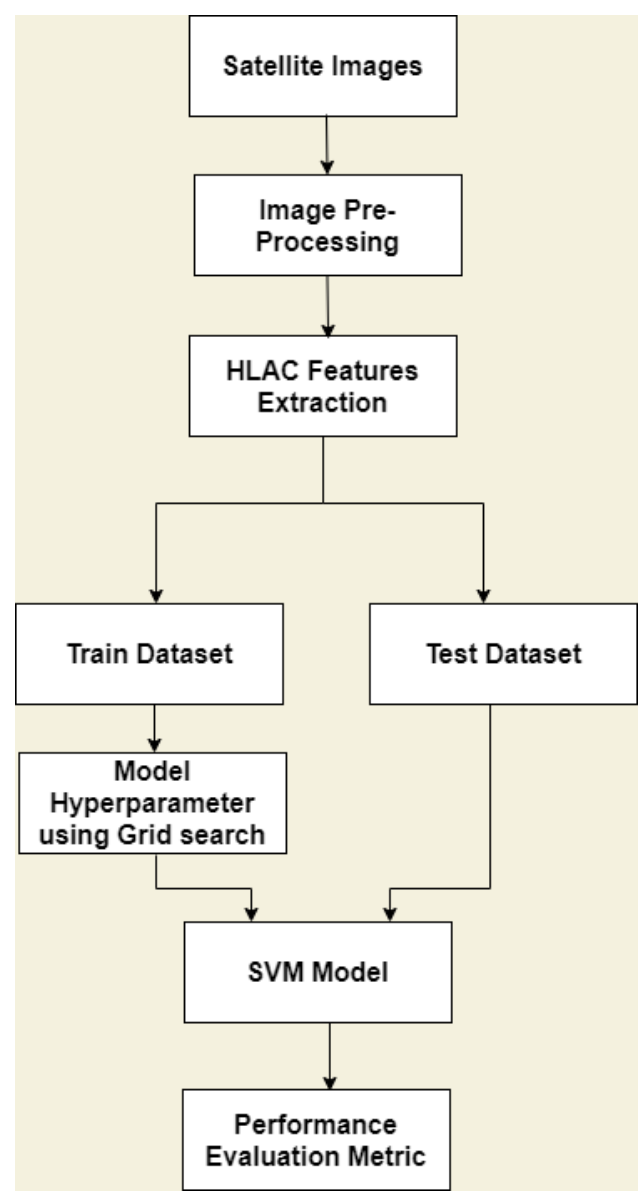

Fig.1. Satellite image classification using HLAC: Proposed method

Figure 1 illustrates the suggested technique's workflow. First and foremost, satellite images from various classes are provided as input. The images are of 64x64 pixels in size and all of the images are colored. Following that, all color images are transformed to binary images as a preprocessing step. HLAC features provided by Otsu [Otsu and Kurita (1988)] were used to extract features in this study. Features in HLAC are meant to recognize features in binary images. The dataset is separated into train and test sections after feature extraction. The division is done on a class-by-class basis. The classifier receives the training dataset as input.

SVM uses a one-vs-all strategy to train each class individually for multi-class classification. The grid search method is used to find the model parameters of each class. The SVM model is thus trained throughout the full dataset. Following that, test images are used to check the classifier's performance. 


\subsection{Satellite Image dataset}

This study used the EuroSAT dataset, which was proposed by Helber et al. [Helber et al. (2019)]. This dataset is used to solve the problem of land use and land cover. Images from the Sentinel-2 satellite are included in this dataset. The RGB and Multispectral picture file formats are also supported by the dataset. There are 27,000 photos in ten different classes in total. The classification accuracy of SIFT (BoVW), shallow CNN (2 layers), and deep CNN was compared by the authors (pre-trained models like ResNet-50 and GoogLeNet). With training and testing splits of $80 / 20$, the maximum accuracy was around $70.05 \%$ using handmade feature learning approaches like SIFT (BoVW, $\mathrm{k}=500)$, where $\mathrm{k}$ denotes k-means clustering.

\subsection{Image pre-processing}

All color images are separated into three bands: R, G, and B. Each band is then transformed into binary images for HLAC feature extraction.

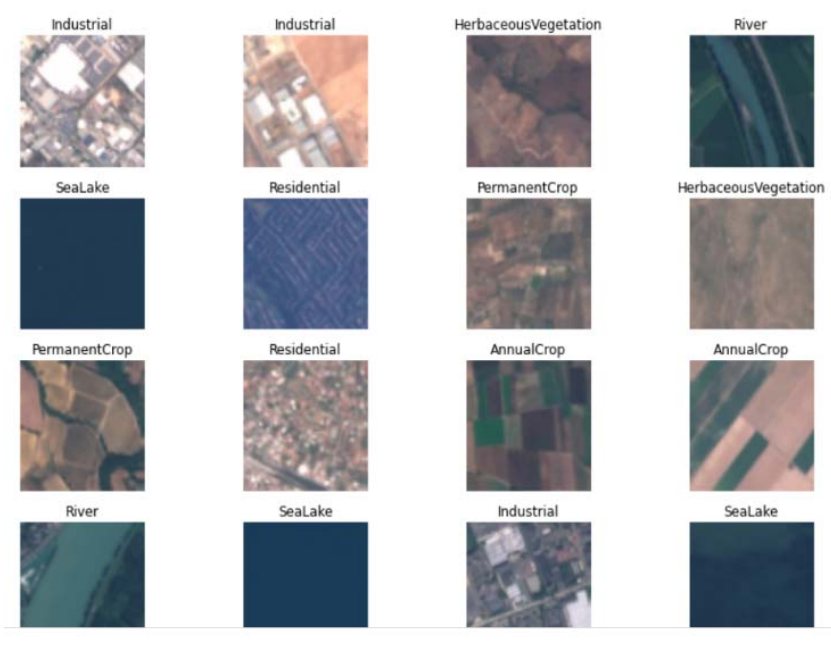

Fig.2. Sample image patches of 10 classes of EuroSAT dataset [Helber et al. (2018)]

\subsection{Feature Extraction}

Image features must be extracted from the given images to classify the satellite images. The satellite images are more texture-like; hence HLAC features were employed to extract features from the image datasets in this research. Otsu and Kurita proposed the HLAC in 1988 [Otsu and Kurita (1988)]. It was capable of capturing shift-invariant and additive geometric characteristics. In addition, because the characteristics can be computed using product sum operations, the HLAC is quick enough to extract them. Object detection [Uehara et al. (2017)], color image feature extraction [Kobayashi and Otsu (2009)], facial feature recognition [Bulugu and Banzi (2017)], and gesture recognition [Ishihara and Otsu (2004), Kurita and Hayamizu (1998)] were all surpassed by the HLAC.

The nth-order auto-correlation function can be defined as Eq.1,

$$
x\left(d_{1}, d_{2}, \ldots, d_{N}\right)=\int f(p)+f\left(p+d_{1}\right)+\cdots+f\left(p+d_{N}\right)
$$

Where, $d_{1}, d_{2} \ldots d_{N}$ are $N$ displacements, and $f(p)$ is the intensity of the reference pixel at point $r$.

Figure 3 shows the HLAC mask patterns for a $3 \times 3$ mask size. There are a total of 35 different mask combinations if the order is limited to two. In each mask, the black color symbolizes "needed" or reference points, while the white color denotes "not required" points. To make the scale of the feature invariant, mask patterns of different sizes are employed. Here, we have used $3 \times 3,5 \times 5$, and $7 \times 7$ mask patterns as shown in figure 4. 


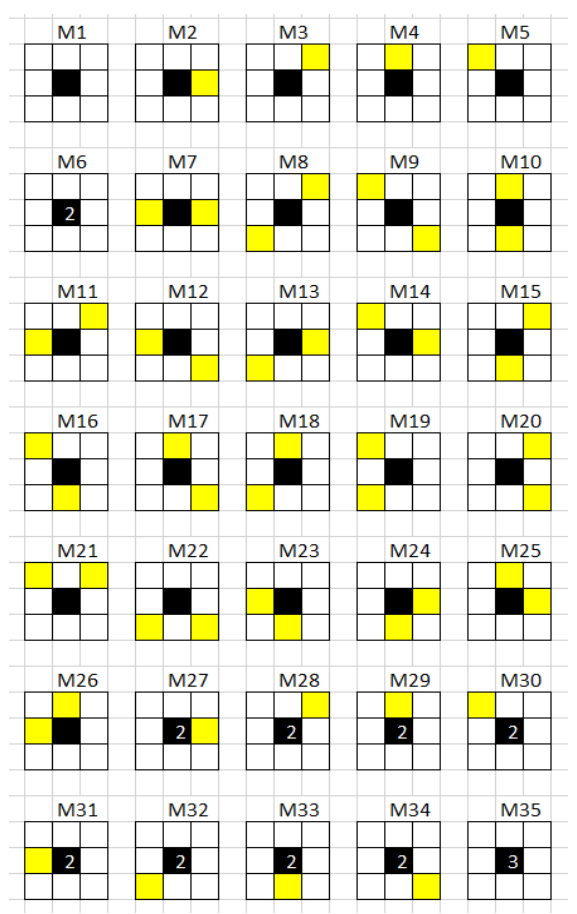

Fig.3. HLAC mask patterns for $3 \times 3$ mask size [Uehara (2017)]

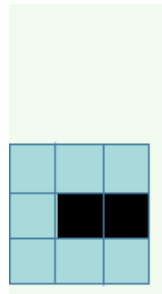

$3 \times 3$ mask

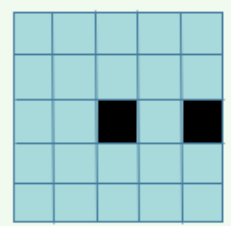

$5 \times 5$ mask

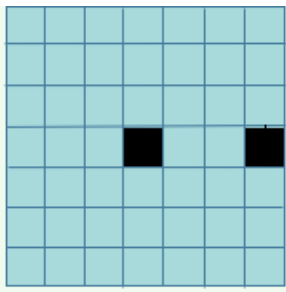

$7 \times 7$ mask

Fig.4. HLAC mask patterns for different mask size

\subsection{Support Vector Machine (SVM) Classifier}

Since 1963, the SVM has been one of the most used supervised binary classification techniques. Cortes and Vapnik [Cortes and Vapnik (1995)] introduced support vector networks in 1995 for two-class classification problems. Vladimir Vapnik [Vladimir Vapnik (1998)] proposed the SVM as a supervised learning technique. Text and image classification [Russakovsky et al. (2015)], oil tank identification [Jivane and Soundrapandiyan (2017)], medical analysis [Battineni et al. (2019)], and stock market trading [Deepak et al.(2017)] are just a few of the real-world implementations for SVM. SVM is also known as a large margin classifier since it optimizes the margins of the separating hyperplanes. Because satellite images are texture images, SVM performs well.

For a small dataset, SVM performs better, but it is time-consuming for a large dataset. SVM uses kernel functions to divide non-separable data into a higher-dimensional space.

\subsection{Evaluation Criterion}

The accuracy and F1-score are used to evaluate the classifier's performance. The accuracy of a dataset is calculated by dividing the total number of correct predictions by the total number of predictions. The harmonic mean of precision and recall are calculated using the F1-score mathematical equation. 


\section{Image Classification using Deep Learning Features (Deep CNNs)}

We separated the data for each dataset into a training and a test set at a ratio of $80 / 20$ to train and evaluate deep CNNs on the proposed innovative and introduced existing datasets. We made sure the split was applied to each class separately. Except for the typical vertical flipping, we used no data augmentation in our studies. We finetuned ResNet-50 and ResNet-101 [He et al.(2016), He et al.(2016)] CNN models for each dataset, which were pre-trained on the ILSVRC-2012 image 205 classification dataset. We have trained the last layer using a learning rate of $0.001 \mathrm{in}$ all evaluations. Then, using a modest learning rate of $10^{-6}$ to $10^{-5}$, we fine-tuned the entire network. To evaluate the performance of the different CNN models on the analyzed datasets, we computed the total classification accuracy.

\section{Results and Discussion}

The EuroSAT dataset was utilized as the basis for this investigation. Here, all 10 classes were taken into account. Each class has between 2000 and 3000 labeled images. As a result, the experiment used a total of 27,000 images. The features are extracted from the images using HLAC. Various kernel functions, such as the linear kernel, polynomial kernel, and RBF kernels, were used. The grid search algorithm was used to select the various kernel parameters to improve the results. For multi-class classification, SVM employs a one-vs-all classification technique. The accuracy and F1-score criteria are used to assess the performance. We have used pre-trained models ResNet-50 and ResNet-101 were trained on the EuroSAT dataset. To test the model's performance on the EuroSAT dataset, we evaluated the total classification accuracy.

\begin{tabular}{|c|c|c|c|c|c|}
\hline Classifier & $\begin{array}{c}\text { Method } \\
\text { used }\end{array}$ & $\begin{array}{c}\text { Performance } \\
\text { Evaluation } \\
\text { Criteria }\end{array}$ & \multicolumn{3}{|c|}{$\begin{array}{c}\text { Classification Accuracy (\%) with } \\
\text { Training/Testing split 80/20 }\end{array}$} \\
\hline $\begin{array}{c}\text { SVM } \\
\text { (benchmark } \\
\text { results) }\end{array}$ & $\begin{array}{c}\text { SIFT } \\
\text { (BoVW) }\end{array}$ & $\begin{array}{c}\text { Accuracy } \\
(\%)\end{array}$ & $\begin{array}{c}58.55 \\
(\mathrm{k}=10)\end{array}$ & $\begin{array}{c}67.22 \\
(\mathrm{k}=100\end{array}$ & $\begin{array}{c}70.05 \\
(\mathrm{k}=500)\end{array}$ \\
\hline \multirow{2}{\text{SVM}}{$\begin{array}{c}\text { (results of } \\
\text { proposed } \\
\text { approach) }\end{array}$} & HLAC & Accuracy & $\begin{array}{c}87.84 \\
(\%)\end{array}$ & $\begin{array}{c}88.35 \\
(\mathrm{RBF})\end{array}$ & $\begin{array}{c}89.35 \\
\text { (Poly) }\end{array}$ \\
\cline { 3 - 6 } & & F1-score & $\begin{array}{c}85.37 \\
\text { (RBF) }\end{array}$ & $\begin{array}{c}86.14 \\
\text { (Lin) }\end{array}$ & $\begin{array}{c}86.26 \\
\text { (Poly) }\end{array}$ \\
\hline
\end{tabular}

Table 1. Comparison of classification accuracy of handcrafted features with benchmark

Table 1 shows the classification accuracy and F1-score for 10 classes in the EuroSAT dataset. The outcomes were compared to the benchmarks established in [Helber et al. (2019)]. With a train and test splitting ratio of $80 / 20$ and the SIFT (BoVW) technique, the optimum classification accuracy with SVM was $70.05 \%$ in the benchmark. Using HLAC feature extraction, the SVM classifier was able to efficiently distinguish between separate classes, improving accuracy to $89.35 \%$ with a train and test splitting ratio of $80 / 20$. The accuracy has improved by $19.3 \%$ with the benchmark.

To improve accuracy, many kernel methods were utilized, including the linear kernel, polynomial kernel, and RBF kernel. The RBF kernel had the lowest accuracy (87.84\%), the linear kernel was in the middle $(88.35 \%)$, and the polynomial kernel had the best accuracy $(89.35 \%)$. The minimum F1-score for the RBF kernel was 85.37, and the maximum F1-score for the polynomial kernel was 86.26. Figure 5 additionally depicts the accuracy of the reported result vs. the benchmark as a graph. 


\section{Accuracy(\%)}

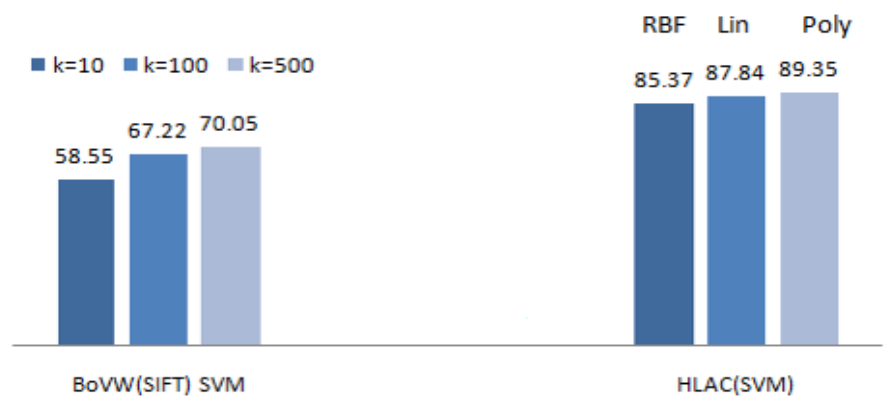

Fig.5. Comparison of classification accuracy

The classification results for the various deep CNN models are listed in Table 2. The authors in [Helber et al. (2019)] employed a pre-trained ResNet-50 model [He et al. (2016), He et al. (2016)] that was trained on the EuroSAT dataset and attained a classification accuracy of $98.57 \%$. We utilized a pre-trained ResNet-50 and ResNet-101 model and trained on the EuroSAT dataset and attained an accuracy of $98.69 \%$. On the EuroSAT dataset [Helber et al. (2018), Helber et al. (2019)], the deep CNNs produce state-of-the-art results, outperforming earlier results by $0.12 \%[15,20]$. Figure 6 depicts the confusion matrix of this best-performing network, which allows for a class-level evaluation. The classifier occasionally confuses the River and Highway classes, the AnnualCrop and PermanentCrop classes, as well as the PermanentCrop and HerbaceousVegetation classes even if this occurs infrequently.

\begin{tabular}{|c|c|}
\hline Method & Accuracy (\%) \\
\hline ResNet-50 (benchmark) & 98.57 \\
\hline ResNet-50 (our result) & 98.50 \\
\hline ResNet-101 (our result) & 98.69 \\
\hline
\end{tabular}

Table 2. Comparison of classification accuracy of deep learning models with benchmark

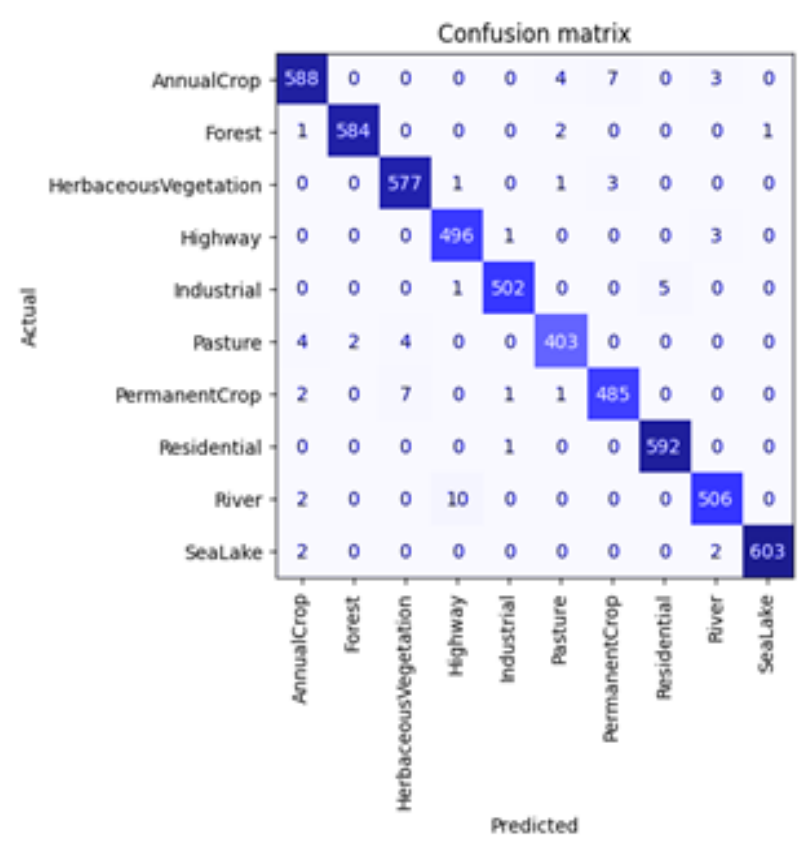

Fig.6. Confusion matrix of ResNet-101 model 


\section{Conclusion and Future Work}

In this study, we compared various models based on deep learning, transfer learning, and models trained on hand-crafted features. HLAC features were used with the SVM classifier to classify satellite images. SVM provided good classification accuracy using the HLAC features extraction methods. The increased accuracy of the SVM classifier showed that it could effectively distinguish between distinct classes when features were extracted using HLAC. Using other kernels and altering their settings improves the outcomes much more. Using a polynomial kernel, the accuracy was improved by $19.3 \%$ for ten classes.

The ResNet-50 and ResNet-101 models were used to evaluate the performance of deep learning features, and the performance was enhanced by $0.12 \%$ using the ResNet- 101 model compared with benchmark results.

In future work, the order of the HLAC features might be increased to improve the accuracy. Furthermore, by evaluating all of the spectral bands of the satellite images, the results can be enhanced. To improve classification, handcrafted features can be merged with deep learning models.

\section{References}

[1] Battineni, G., Chintalapudi, N., Amenta, F. (2019). Machine learning in medicine: Performance calculation of dementia prediction by support vector machines (SVM). Informatics in Medicine Unlocked, 16, 100200.

[2] Bulugu,I.; Ye, Z.; Banzi,J. (2017): Higher-order local autocorrelation feature extraction methodology for hand gestures recognition, In 2017 2nd International Conference on Multimedia and Image Processing (ICMIP), pp. 83-87.

[3] Cao, J., Cholakkal, H., Anwer, R. M., Khan, F. S., Pang, Y., \& Shao, L. (2020). D2det: Towards high quality object detection and instance segmentation. In Proceedings of the IEEE/CVF conference on computer vision and pattern recognition (pp. 11485-11494).

[4] Cortes, C.; Vapnik, V. (1995): Support-vector networks, Machine learning 20, no. 3, pp. 273-297.

[5] Csurka, G.; Dance C.R.; Humenberger M. (20.18): From handcrafted to deep local features, arXiv preprint arXiv: 1807.10254, 2018.

[6] Dalal, N. ; Triggs, B. (2005): Histograms of oriented gradients for human detection, In 2005 IEEE computer society conference on computer vision and pattern recognition (CVPR'05), vol. 1, pp. 886-893.

[7] Deepak, R. S., Uday, S. I., \& Malathi, D. (2017). Machine learning approach in stock market prediction. International Journal of Pure and Applied Mathematics, 115(8), 71-77.

[8] Dewangkoro, H. I.; Arymurthy, A. M. (2021): Land Use and Land Cover Classification Using CNN, SVM, and Channel Squeeze \& Spatial Excitation Block, In IOP Conference Series: Earth and Environmental Science, IOP Publishing, vol. 704, no. 1, pp. 012048.

[9] Haralick, R. M.; Shanmugam, K.; and Dinstein, H. (1973): Textural features for image classification, IEEE Transactions on systems, man, and cybernetics, vol.3, no.6, pp. 610-621.

[10] Hayashi, H. ; Uchida, S. (2018): A trainable multiplication layer for auto-correlation and co-occurrence extraction," In: Asian Conference on Computer Vision, Springer, pp. 414-430.

[11] He, K., Zhang, X., Ren, S., \& Sun, J. (2016). Deep residual learning for image recognition. In Proceedings of the IEEE conference on computer vision and pattern recognition (pp. 770-778).

[12] He, K., Zhang, X., Ren, S., \& Sun, J. (2016). Identity mappings in deep residual networks. In European conference on computer vision (pp. 630-645). Springer, Cham.

[13] Helber, P., Bischke, B., Dengel, A., \& Borth, D. (2018, July). Introducing eurosat: A novel dataset and deep learning benchmark for land use and land cover classification. In IGARSS 2018-2018 IEEE International Geoscience and Remote Sensing Symposium, pp. 204-207.

[14] Helber, P., Bischke, B., Dengel, A., \& Borth, D. (2019). Eurosat: A novel dataset and deep learning benchmark for land use and land cover classification. IEEE Journal of Selected Topics in Applied Earth Observations and Remote Sensing, 12(7), $2217-2226$.

[15] Humeau-Heurtier, A. (2019). Texture feature extraction methods: A survey. IEEE Access, 7, 8975-9000.

[16] Ishihara,T.; Otsu, N.(2004): Gesture recognition using auto-regressive coefficients of higher-order local auto-correlation features," In: Sixth IEEE International Conference on Automatic Face and Gesture Recognition Proceedings, pp. 583-588.

[17] Jivane, N.J. ; Soundrapandiyan, R. (2017): Enhancement of an algorithm for oil tank detection in satellite images, International Journal of Intelligent Engineering System, vol. 10, no.3, pp. 298-305.

[18] Kobayashi, T. ; Otsu, N. (2009): "Color image feature extraction using color index local auto-correlations," In: 2009 IEEE International Conference on Acoustics, Speech and Signal Processing, pp. 1057-1060.

[19] Kupidura, P. (2019): The comparison of different methods of texture analysis for their efficacy for land use classification in satellite imagery, Remote Sensing, vol. 11, issue 10, pp. 1233.

[20] Kurita T.; Hayamizu, S. (1998): Gesture recognition using hlac features of parcor images and hmm based recognizer, In: Proceedings Third IEEE International Conference on Automatic Face and Gesture Recognition, pp. 422-427.

[21] Kussul, N., Shelestov, A., Lavreniuk, M., Butko, I., \& Skakun, S. (2016). Deep learning approach for large scale land cover mapping based on remote sensing data fusion. In 2016 IEEE International Geoscience and Remote Sensing Symposium (IGARSS) (pp. 198201). IEEE.

[22] Li, K., Wan, G., Cheng, G., Meng, L., \& Han, J. (2020). Object detection in optical remote sensing images: A survey and a new benchmark. ISPRS Journal of Photogrammetry and Remote Sensing, 159, 296-307.

[23] Liu, Q.; Basu S.; Ganguly, S.; Mukhopadhyay, S.; DiBiano, R.; Karki, M.; Nemani, R. (2020): Deepsat v2: feature augmented convolutional neural nets for satellite image classification, Remote Sensing Letters, vol.11, no. 2, pp. 156-165.

[24] Lowe, D.G. (2004): Distinctive image features from scale-invariant keypoints, International journal of computer vision vol. 60 no.), pp. 91-110.

[25] Noi, P. T.; Kappas, M. (2018): Comparison of random forest, k-nearest neighbor, and support vector machine classifiers for land cover classification using sentinel-2 imagery, Sensors, vol .18(1).

[26] Oh, S. I., \& Kang, H. B. (2017). Object detection and classification by decision-level fusion for intelligent vehicle systems. Sensors, $17(1), 207$.

[27] Otsu, N.; Kurita, T. (1988): A new scheme for practical flexible and intelligent vision systems, In: MVA, pp. $431-435$.

[28] Quinlan, J.R. (1986): Induction of decision trees, Machine learning, vol. 1, no.1, pp. 81-106. 
[29] Rana V.K.; Suryanarayana, T.M.V.(2020): Performance evaluation of mle, rf and svm classification algorithms for watershed scale land use/land cover mapping using sentinel 2 bands, Remote Sensing Applications: Society and Environment, vol 19, pp. 100351.

[30] Russakovsky, O; Deng, J.; Su, H.; Krause, J.; Satheesh, S.; Ma, S.; Huang, Z.; Karpathy, A.; Khosla, A.; Bernstein, M.; Berg, A. C.; Fei-Fei, L. (2015): ImageNet Large Scale Visual Recognition Challenge, International Journal of Computer Vision (IJCV), vol. 115, no. 3, pp. 211-252.

[31] Tianyu, Z.; Zhenjiang, M.; Jianhu, Z. (2018): Combining cnn with hand-crafted features for image classification, In: 2018 14th IEEE International Conference on Signal Processing (ICSP 2018), pp. 554-557.

[32] Uehara, K. ; Sakanashi, H.; Nosato, H.; Murakawa, M.; Miyamoto, H.; Nakamura, R. (2017): Object detection of satellite images using multi-channel higher-order local autocorrelation, In: 2017 IEEE International Conference on Systems, Man, and Cybernetics (SMC), IEEE, pp. 1339-1344.

[33] Vapnik, V.(1998): Statistical learning theory New York, NY: Wiley.

[34] Varma, M.K.S. ; Rao, N.K. (2017): Satellite image classification using genetic algorithm based on svm classifier, International Journal of Control Theory and Applications, vol. 10, no.26, pg. 12-20.

[35] Zhang, X.; Cui, J. ; Wang, W.; Lin, C. (2017): A study for texture feature extraction of high-resolution satellite images based on a direction measure and gray level co-occurrence matrix fusion algorithm, Sensors vol. 17(7), pg. 1474.

[36] Zhong, Y., Fei, F., Liu, Y., Zhao, B., Jiao, H., \& Zhang, L. (2017). SatCNN: satellite image dataset classification using agile convolutional neural networks. Remote sensing letters, 8(2), 136-145.

\section{Authors Profile}
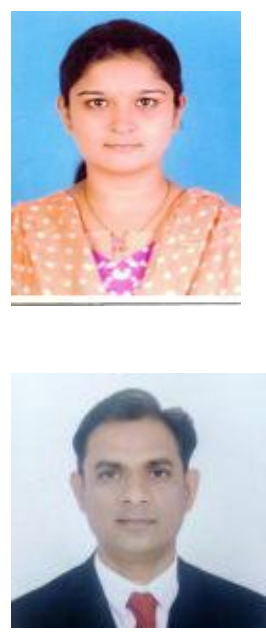

Khushbu R. Joshi is a research scholar in the department of Sankalchand Patel University Visnagar, India. She is also working as a lecturer in the Electronics and communication department of LDRP Institute of Technology and Research, Gandhinagar, India. She obtained her BE degree in Electronics and Communication Engineering in 2007 from Saurashtra University, Rajkot and ME degree in Electronics and Communication (CSE) in 2009 from Gujarat University, Ahmedabad. Her research interests include Machine Learning, Deep Learning and Computer Vision System. She is a member of IETE, India.

Manish I. Patel is working as an Assistant Professor in Electronics and Communication Engineering Department since November 2019. He has more than 15 years of teaching experience. He obtained his $\mathrm{BE}$ degree in Electronics and Communication Engineering in 2003 from North Gujarat University, Patan and MTech degree in Electronics and Communication (VLSI Design) in 2010 from Nirma University, Ahmedabad. Dr Manish obtained his PhD in 2017 from Gujarat Technological University, Ahmedabad. His areas of interest include signal and image processing, machine learning and VLSI design. He has published more than fifteen papers in journals and conference proceedings. He has guided PG students. He is a life member of ISTE and IETE, India. 